\title{
A nonexistence of solutions to a supercritical problem
}

\author{
Kamal Ould Bouh \\ Department of Mathematics, Taibah University, Almadinah Almunawwarah, KSA
}

Email addresses:

hbouh@taibahu.edu.sa,kamal_bouh@yahoo.fr

\section{To cite this article:}

Kamal Ould Bouh. A Nonexistence of Solutions to a Supercritical Problem. Pure and Applied Mathematics Journal. Vol. 2, No. 6, 2013, pp. 184-190. doi: 10.11648/j.pamj.20130206.13

\begin{abstract}
In this paper, we study the nonlinear elliptic problem involving nearly critical exponent $\left(P_{\epsilon}\right):-\Delta u=$ $K u^{\frac{n+2}{n-2}+\epsilon}$ in $\Omega ; u>0$ in $\Omega$ and $u=0$ on $\partial \Omega$ where $\Omega$ is a smooth bounded domain in $I^{n}, n \geq 3, K$ is a $C^{3}$ positive function and $\epsilon$ is a small positive real parameter. We prove that, for $\mathcal{E}$ small, $\left(P_{\varepsilon}\right)$ has no positive solutions which blow up at one critical point of the function $K$.
\end{abstract}

Keywords: Nonlinear Elliptic Equations, Critical Exponent, Variational Problem

\section{Introduction}

Let $\Omega$ be a smooth bounded domain in $\mathbb{R}^{n}, n \geq 3$. We consider the following nonlinear elliptic problem

$$
\left(P_{\epsilon}\right)\left\{\begin{array}{lr}
-\Delta u=K u^{p+\epsilon} & \text { in } \Omega \\
u>0 & \text { in } \Omega \\
u=0 & \text { on } \partial \Omega
\end{array}\right.
$$

where $K$ is a $C^{3}$ positive function, $p+1=2 n / n-2$ is the critical Sobolev exponent and $\epsilon$ is a small positive real parameter.

Problem $\left(P_{\epsilon}\right)$ is in some sense related to the limiting problem(when $\square=0$ ) and the interest to it comes from its resemblance to the scalar curvature problem in differential geometry, which consists in finding suitable conditions on a given function $K$ defined on $M$ such that $K$ is the scalar curvature for a metric $\bar{g}$ conformally equivalent to $g$, where $(M, g)$ is a $\mathrm{n}$-dimensional Riemannian manifold without boundary.

Note that the limiting problem has bee1.5

$\mathrm{n}$ widely studied in various works see for example [1], [2], [7] and [10].

In another view point, it is interesting to study the problem $\left(P_{\epsilon}\right)$ with $\square<0$ and $\square>0$ and to understand what happens to the solutions of $\left(P_{\epsilon}\right)$ (if they exist) as $\square \rightarrow 0$ !!

When $\square \in(1-p, 0)$, the mountain pass lemma proves the existence of solutions of $\left(P_{\epsilon}\right)$ (see [3]). Note that, many works have been devoted to the study of positive solutions of $\left(P_{\epsilon}\right)$ with $\square<0$. In sharp contrast to this, very little study has been made concerning the sign-changing solutions of $\left(P_{\epsilon}\right)$ with $\square<0$ and even less for $\square>0$.
When $\square>0$, problem $\left(P_{\epsilon}\right)$ becomes more delicate since we loose the Sobolev embedding which is an important difficulty to overcome.

Concerning the supercritical case, $\square>0$ and $K$ is a constant, it was proved in [4] that $\left(P_{\epsilon}\right)$ has no positive solution which blows up at a single point. This result shows that the situation is different from the subcritical one. However, del Pino et al [6] gave an existence result for two blow up points, provided that $\Omega$ satisfies some geometrical conditions. In sharp contrast to this, it proved in [5] for the case $K$ is a constant and [8] for the case $K$ is a non constant function that, for $\square$ small, $\left(P_{\epsilon}\right)$ has no sign-changing solutions which blow up at two points.

In this paper, we consider the case $K$ is a non constant function and we look to understand the influence of the function $K$ in the study of the positive solutions of $\left(P_{\epsilon}\right)$ which blows up at a single point.

It is well known that problem $\left(P_{\epsilon}\right)$ has a variational structure. Setting

$J(u)=\frac{\int_{\Omega}|\nabla u|^{2}}{\left(\int_{\Omega} K|u|^{p+1+\epsilon}\right)^{\frac{2}{p+1+\epsilon}}}, u \in H_{0}^{1}(\Omega), u \neq \equiv 0$,

the positive critical points of $J$ are solutions to $\left(P_{\epsilon}\right)$, up to a multiplicative constant. $J$ satisfies the Palais-Smale condition in the subcritical case, whereas this condition fails in the critical case. Such a failure is due to the function

$\delta_{(a, \lambda)}(x)=C_{0} \frac{\lambda^{\frac{n-2}{2}}}{\left(1+\lambda^{2}|x-a|^{2}\right)^{\frac{n-2}{2}}}, C_{0}=(n(n-2))^{\frac{n-2}{4}}, \lambda>0, a \in \mathbb{R}^{n}$

which are the only solutions of

$-\Delta u=u^{\frac{n+2}{n-2}}, u>0$ in $\mathbb{R}^{n}$, with $u \in L^{p+1}\left(\mathbb{R}^{n}\right)$ and $\nabla u \in L^{2}\left(\mathbb{R}^{n}\right)$ and are also the only minimizers of the 
Sobolev inequality on the whole space, that is

$$
\left.S=|u|_{\frac{2 n}{n-2}\left(\mathbb{R}^{n}\right)}^{-2}, s . t \nabla u \in L^{2}, u \in L^{\frac{2 n}{n-2}}\left(\mathbb{R}^{n}\right), u \neq 0\right\}
$$

We have the following nonexistence result for $\left(P_{\epsilon}\right)$ :

\section{Theorem 1}

Let $\Omega$ be any smooth bounded domain in $\mathbb{R}^{n}, n \geq 3$. Assume that $a_{0} \in \Omega$ is a critical point of $K$ satisfying one of the following conditions:

(i) $n=3$,

(ii) $n=4$, and $c_{1} H\left(a_{0}, a_{0}\right)-\frac{c_{3} \Delta K\left(a_{0}\right)}{16 K\left(a_{0}\right)}>0$,

(iii) $n \geq 5$, and $-\Delta K\left(a_{0}\right)>0$.

Then the problem $\left(P_{\epsilon}\right)$ has no solution $u_{\epsilon}$ such that $u_{\epsilon}=\alpha_{\epsilon} P \delta_{a_{\epsilon}, \lambda_{\epsilon}}+v_{\epsilon}$ with $\left|u_{\epsilon}\right|^{\epsilon}$ is bounded and

$v_{\epsilon} \rightarrow 0$ in $H_{0}^{1}(\Omega) \alpha_{\epsilon} \rightarrow K(a)^{(2-n) / 4}, a_{\epsilon} \in \Omega, a_{\epsilon} \rightarrow$ $a_{0}$ and $\lambda_{\epsilon} d\left(a_{\epsilon}, \partial \Omega\right) \rightarrow+\infty$ as $\epsilon \rightarrow 0$.

\section{Preliminary Results}

We need to introduce some notations:

$P \delta_{a, \lambda}$ is defined as the only function in $H_{0}^{1}(\Omega)$ such that $\Delta P \delta_{a, \lambda}=\Delta \delta_{a, \lambda}$. Writing

$$
P \delta_{a, \lambda}=\delta_{a, \lambda}-\theta_{a, \lambda}
$$

we have

$$
\Delta \theta_{a, \lambda}=0 \text { in } \Omega ; \theta_{a, \lambda}=\delta_{a, \lambda} \text { on } \partial \Omega
$$

We note that projections $P \delta_{a, \lambda}$ of $\delta_{a, \lambda}$ 's on $H_{0}^{1}(\Omega)$ are approximate solutions to the limiting problem as $a_{\epsilon} \in \Omega$ and $\lambda_{\epsilon} d\left(a_{\epsilon}, \partial \Omega\right)$ goes to infinity.

Let $G$ be the Green's function for the Laplace operator with Dirichlet boundary conditions, that is, for any $x \in \Omega$.

$$
\left\{\begin{array}{c}
-\Delta G(x, .)=c_{n} \delta_{x} \text { in } \Omega \\
G(x, .)=0 \text { on } \partial \Omega
\end{array}\right.
$$

with $\delta_{x}$ the Dirac mass at $x$ and $c_{n}=(n-2)\left|S^{n-1}\right|$

We denote by $H$ the regular part of $G$, i.e .

$$
\begin{gathered}
H\left(x_{1}, x_{2}\right)=\left|x_{1}-x_{2}\right|^{2-n}-G\left(x_{1}, x_{2}\right) \\
\text { for }\left(x_{1}, x_{2}\right) \in \Omega \times \Omega
\end{gathered}
$$

The maximum principle provides us with the uniform estimate

$\theta_{a, \lambda}(x)=C_{0} \frac{H(x, a)}{\lambda^{\frac{n-2}{2}}}+O\left(\frac{1}{\lambda^{\frac{n+2}{2}}(d(a, \partial \Omega))^{n}}\right) \operatorname{as} \lambda d(a, \partial \Omega) \rightarrow+\infty$

Corresponding estimates hold for the derivatives of $\theta_{a, \lambda}$ with respect to $a, \lambda$ and $x$.

Note that $H(x, x)=O\left(d(x, \partial \Omega)^{2-n}\right)$ as $d(x, \partial \Omega) \rightarrow 0$ [9]. From [9] we also know that

$\int_{\Omega}\left|\nabla \theta_{a, \lambda}\right|^{2}=O\left(\lambda d(a, \partial \Omega)^{2-n}\right) \operatorname{as} \lambda d(a, \partial \Omega) \rightarrow+\infty$
Next, we recall that for $u_{\epsilon}$ satisfying the assumption of the theorem, there is a unique way to choose $a_{\epsilon}, \lambda_{\epsilon}$ and $v_{\epsilon}$ such that

$$
u_{\epsilon}=\alpha_{\epsilon} P \delta_{a_{\epsilon}, \lambda_{\epsilon}}+v_{\epsilon}
$$

with

$$
\left\{\begin{array}{c}
\alpha_{\epsilon} \in \mathbb{R}, \quad \alpha_{\epsilon} \rightarrow K\left(\alpha_{\epsilon}\right)^{(2-n) / 4} \\
a_{\epsilon} \in \Omega, \lambda_{\epsilon} \in \mathbb{R}_{+}^{*}, \lambda_{\epsilon} d\left(a_{\epsilon}, \partial \Omega\right) \rightarrow+\infty \\
v_{\epsilon} \rightarrow 0 \text { in } H_{0}^{1}(\Omega), v_{\epsilon} \in E_{a_{\epsilon}, \lambda_{\epsilon}}
\end{array}\right.
$$

and for any $(a, \lambda) \in \Omega \times \mathbb{R}_{+}^{*}, E_{(a, \lambda)}$ denotes the subspace of $H_{0}^{1}(\Omega)$ defined by

$E_{(a, \lambda)}=\left\{w \in H_{0}^{1}(\Omega) /\left(\mathrm{w}, P \delta_{(a, \lambda)}\right)_{H_{0}^{1}}=\left(w, \frac{\partial P \delta_{(a, \lambda)}}{\partial \lambda}\right)_{H_{0}^{1}}\right.$

$\left.=\left(w, \frac{\partial P \delta_{(a, \lambda)}}{\partial a_{i}}\right)_{H_{0}^{1}}=0,1 \leq i \leq n\right\}$

For the proof of this fact, see [1], [9]. In the following, we always assume that $u_{\epsilon}$, satisfying the assumption of the theorem, is written as in (8). In order to simplify the notations, we set

$$
\delta_{a_{\epsilon}, \lambda_{\epsilon}}=\delta_{\epsilon}, P \delta_{a_{\epsilon}, \lambda_{\epsilon}}=P \delta_{\epsilon} \text { and } \theta_{a_{\epsilon}, \lambda_{\epsilon}}=\theta_{\epsilon}
$$

Lemma 2

Let $u_{\epsilon}$ satisfying the assumption of the theorem 1 . Then

(i) $\int_{\Omega}\left|\nabla u_{\epsilon}\right|^{2} \rightarrow S^{n / 2} \quad ; \quad$ (ii) $\int_{\Omega} K u_{\epsilon}^{p+1+\epsilon} \rightarrow S^{n / 2}$

as $\epsilon \rightarrow o, S, \mathrm{~S}$ denoting the Sobolev constant defined by (2).

\section{Proof.}

We have

$\int_{\Omega}\left|\nabla u_{\epsilon}\right|^{2}=\int_{\Omega}\left|\nabla\left(\alpha_{\epsilon} P \delta_{\epsilon}+v_{\epsilon}\right)\right|^{2}=\alpha_{\epsilon}{ }^{2} \int_{\Omega}\left|\nabla P \delta_{\epsilon}\right|^{2}+$ $\int_{\Omega}\left|\nabla v_{\epsilon}\right|^{2}$ since $v_{\epsilon} \in E_{a_{\epsilon}, \lambda_{\epsilon}}$

From the fact that $\delta_{\epsilon}$ satisfies $-\Delta \delta_{\epsilon}=\delta_{\epsilon}^{P}$ in $\mathbb{R}^{n}$ and is a minimizer for $S$, we deduce that $\int_{\mathbb{R}^{n}}\left|\nabla \delta_{\epsilon}\right|^{2}=S^{n / 2}$

On the other hand, an explicit computation provides us with

$$
\begin{gathered}
\int_{\Omega}\left|\nabla \delta_{a, \lambda}\right|^{2}=\int_{\mathbb{R}^{n}}\left|\nabla \delta_{a, \lambda}\right|^{2}+O\left(\frac{1}{(\lambda d(a, \partial \Omega))^{n}}\right) \text { as } \lambda d(a, \partial \Omega) \\
\rightarrow+\infty .
\end{gathered}
$$

Taking account of (6), claim (i) is a consequence of (8). Claim (ii) follows from the fact that $u_{\epsilon}$ solves $\left(P_{\epsilon}\right)$.

\section{Estimating $\boldsymbol{v}_{\epsilon}$}

As usual in this type of problems, we first deal with the $\mathrm{v}$-part of $\mathrm{u}$, in order to show that it is negligible with respect to the concentration phenomenon. 


\section{Lemma 3}

Let $u_{\epsilon}$ satisfying the assumption of the theorem. $\lambda_{\epsilon}$ occurring in (7) satisfies

$$
\lambda_{\epsilon}^{\epsilon} \rightarrow 1, \text { as } \quad \epsilon \rightarrow 0
$$

Proof.

According to Lemma 2, we have

$$
\int_{\Omega} K u_{\epsilon}^{p+1+\epsilon}=S^{n / 2}+o(1) \text { as } \quad \epsilon \rightarrow 0
$$

and

$$
\begin{aligned}
& \int_{\Omega} K u_{\epsilon}^{p+1+\epsilon}=\int_{\Omega} K\left(\alpha_{\epsilon} P \delta_{\epsilon}+v_{\epsilon}\right)^{p+\epsilon} \alpha_{\epsilon} P \delta_{\epsilon} \\
& +\int_{\Omega} K u_{\epsilon}^{p+\epsilon} v_{\epsilon} \\
& =\alpha_{\epsilon}^{p+1+\epsilon} \int_{\Omega} K P \delta_{\epsilon}^{p+\epsilon+1} \\
& -\int_{\Omega} \Delta u_{\epsilon} v_{\epsilon} \\
& +O\left(\int_{\Omega} P \delta_{\epsilon}{ }^{p+\epsilon}\left|v_{\epsilon}\right|+\int_{\Omega}\left|v_{\epsilon}\right|^{p+\epsilon} P \delta_{\epsilon}\right) \\
& =\alpha_{\epsilon}{ }^{p+1+\epsilon} \int_{\Omega} K P \delta_{\epsilon}^{p+\epsilon+1} \\
& +O\left(\lambda_{\epsilon}^{\frac{\epsilon(n-2)}{2}} \int_{\Omega} P \delta_{\epsilon}^{p}\left|v_{\epsilon}\right|\right. \\
& \left.+\lambda_{\epsilon}^{\frac{\epsilon(n-2)}{2}} \int_{\Omega}\left|v_{\epsilon}\right|^{p+\epsilon} P \delta_{\epsilon}{ }^{1-\epsilon}+\left|v_{\epsilon}\right|_{H_{0}^{1}}\right) \\
& =\alpha_{\epsilon}^{p+1+\epsilon} \int_{\Omega} K P \delta_{\epsilon}^{p+\epsilon+1} \\
& +O\left(\lambda_{\epsilon}^{\epsilon(n-2) / 2}\left|v_{\epsilon}\right|_{L}^{p+1}\right. \\
& \left.+\lambda_{\epsilon}^{\epsilon(n-2) / 2}\left|v_{\epsilon}\right|_{L^{p+1}}^{p+\epsilon}+\left|v_{\epsilon}\right|_{H_{0}^{1}}\right)
\end{aligned}
$$

Thus

$\int_{\Omega} K u_{\epsilon}^{p+1+\epsilon}=\alpha_{\epsilon}^{p+1+\epsilon} \int_{\Omega} K P \delta_{\epsilon}^{p+\epsilon+1}+o\left(\lambda_{\epsilon}^{\epsilon(n-2) / 2}+1\right)(10)$

We observe that

$\int_{\Omega} K P \delta_{\epsilon}^{p+1+\epsilon}=\int_{\Omega} K\left(\delta_{\epsilon}-\theta_{\epsilon}\right)^{p+\epsilon+1}=\int_{\Omega} K \delta_{\epsilon}^{p+1+\epsilon}+$ $O\left(\int_{\Omega} \delta_{\epsilon}^{p+\epsilon} \theta_{\epsilon}\right)$

$$
\begin{aligned}
& =C_{0}^{p+\epsilon+1} K\left(a_{\epsilon}\right) \int_{\mathrm{B}}\left(\frac{\lambda_{\epsilon}}{1+\lambda_{\epsilon}^{2}\left|x-a_{\epsilon}\right|^{2}}\right)^{\frac{(p+1+\epsilon)(n-2)}{2}}+ \\
& O\left(\left|\theta_{\epsilon}\right|_{L^{\infty}} \int_{\Omega}\left(\frac{\lambda_{\epsilon}}{1+\lambda_{\epsilon}^{2}\left|x-a_{\epsilon}\right|^{2}}\right)^{\frac{(p+1+\epsilon)(n-2)}{2}}+\frac{\frac{\lambda_{\epsilon}(n-2)}{2}}{\left(\lambda_{\epsilon} d_{\epsilon}\right)^{n}}\right)
\end{aligned}
$$

where $B=B\left(a_{\epsilon}, d_{\epsilon}\right)$.Using Proposition 1 of [9], we obtain

$$
\begin{aligned}
& \int_{\Omega} K P \delta_{\epsilon}^{p+1+\epsilon} \\
& =\lambda_{\epsilon}^{\frac{\epsilon(n-2)}{2}} K\left(a_{\epsilon}\right)\left(C_{0}^{p+\epsilon+1} \int_{\mathbb{R}^{n}} \frac{d x}{\left(1+|x|^{2}\right)^{\frac{(p+1+\epsilon)(n-2)}{2}}}\right. \\
& \left.-C_{0}^{p+\epsilon+1} \int_{\mathbb{R}^{n} / B} \frac{d x}{\left(1+|x|^{2}\right)^{\frac{(p+1+\epsilon)(n-2)}{2}}}\right) \\
& +O\left(\frac{\lambda_{\epsilon}^{\frac{\epsilon(n-2)}{2}}}{\left(\lambda_{\epsilon} d_{\epsilon}\right)^{n-2}}\right) \\
& =\lambda_{\epsilon}^{\epsilon(n-2) / 2} K\left(a_{\epsilon}\right) C_{0}^{p+\epsilon+1} \int_{\mathbb{R}^{n}} \frac{d x}{\left(1+|x|^{2}\right)^{(p+1+\epsilon)(n-2) / 2}} \\
& +O\left(\frac{\lambda_{\epsilon}^{\frac{\epsilon(n-2)}{2}}}{\left(\lambda_{\epsilon} d_{\epsilon}\right)^{n-2}}\right)
\end{aligned}
$$

We note that

$$
\begin{gathered}
C_{0}^{p+\epsilon+1} \int_{\mathbb{R}^{n}} \frac{d x}{\left(1+|x|^{2}\right)^{(p+1+\epsilon)(n-2) / 2}}=C_{0}^{p+1} \int_{\mathbb{R}^{n}} \frac{d x}{\left(1+|x|^{2}\right)^{n}}+ \\
O(\epsilon)=S^{n / 2}+O(\epsilon) .
\end{gathered}
$$

Therefore

$$
\int_{\Omega} K P \delta_{\epsilon}^{p+1+\epsilon}=\lambda_{\epsilon}^{\epsilon(n-2) / 2} K\left(a_{\epsilon}\right)\left(S^{n / 2}+O(\epsilon)+o(1)\right.
$$

so (10) and (11) provide us with

$$
\int_{\Omega} K u_{\epsilon}^{p+1+\epsilon}=\alpha_{\epsilon}^{p+1+\epsilon} \lambda_{\epsilon}^{\epsilon(n-2) / 2} K\left(a_{\epsilon}\right)\left(S^{n / 2}+o(1)\right)+
$$

Combination of (9) and (12) proves the lemma.

Next, we recall the following estimate [10] :

\section{Remark 4}

$\delta_{\epsilon}^{\epsilon}(x)-C_{0}^{\epsilon} \lambda_{\epsilon}^{\epsilon(n-2) / 2}=O\left(\epsilon \log \left(1+\lambda_{\epsilon}^{2}\left|x-a_{\epsilon}\right|^{2}\right)\right)$ in $\Omega$. We are now able to study the $v_{\epsilon}$-part of $u_{\epsilon}$.

\section{Lemma 5}

Let $u_{\epsilon}$ satisfying the assumption of the theorem. $v_{\epsilon}$ occurring in (7) satisfies

$$
\leq C+C\left\{\begin{array}{l}
\frac{\nabla K\left(a_{\epsilon}\right)}{\lambda_{\epsilon}}+\frac{1}{\left(\lambda_{\epsilon} d_{\epsilon}\right)^{n-2}} \quad \text { if } n<6 \\
\frac{\nabla K\left(a_{\epsilon}\right)}{\lambda_{\epsilon}}+\frac{\log \left(\lambda_{\epsilon} d_{\epsilon}\right)}{\left(\lambda_{\epsilon} d_{\epsilon}\right)^{4}} \quad \text { if } n=6 \\
\frac{\nabla K\left(a_{\epsilon}\right)}{\lambda_{\epsilon}}+\frac{1}{\left(\lambda_{\epsilon} d_{\epsilon}\right)^{(n+2) / 2}} \quad \text { if } n>6
\end{array}\right.
$$


with $C$ independent of $\epsilon$.

Proof.

Multiplying $\left(P_{\epsilon}\right)$ by $v_{\epsilon}$ and integrating on $\Omega$, we obtain

$$
0=\int_{\Omega} \nabla u_{\epsilon} \cdot \nabla v_{\epsilon}-\int_{\Omega} K u_{\epsilon}^{p+\epsilon} v_{\epsilon}
$$

Thus

$$
\begin{aligned}
0=\int_{\Omega}\left|\nabla v_{\epsilon}\right|^{2}- & \int_{\Omega} K\left[\left(\alpha_{\epsilon} P \delta_{\epsilon}\right)^{p+\epsilon}\right. \\
& +(p+\epsilon)\left(\alpha_{\epsilon} P \delta_{\epsilon}\right)^{p-1+\epsilon} v_{\epsilon} \\
& \left.+O\left(\delta_{\epsilon}^{p-2+\epsilon} v_{\epsilon}^{2} \chi_{\left|v_{\epsilon}\right|<\delta_{\epsilon}}+\left|v_{\epsilon}\right|^{p+\epsilon}\right)\right] v_{\epsilon} .
\end{aligned}
$$

Using the assumption that $\left|u_{\epsilon}\right|^{\epsilon}$ is bounded, we find

$$
0=Q_{\epsilon}\left(v_{\epsilon}, v_{\epsilon}\right)-f_{\epsilon}\left(v_{\epsilon}\right)+O\left(\left|v_{\epsilon}\right|_{H_{0}^{1}}^{\min (3, p+1)}+\left|v_{\epsilon}\right|_{H_{0}^{1}}^{p+1}\right)
$$

with

and

$$
Q_{\epsilon}(v, v)=\left|v_{\epsilon}\right|_{H_{0}^{1}}^{2}-(p+\epsilon) \int K\left(\alpha_{\epsilon} P \delta_{\epsilon}\right)^{p-1+\epsilon} v^{2}
$$

$$
f_{\epsilon}(v)=\int K\left(\alpha_{\epsilon} P \delta_{\epsilon}\right)^{p+\epsilon} v
$$

We observe that

$$
\begin{gathered}
Q_{\epsilon}(v, v)=\left|v_{\epsilon}\right|_{H_{0}^{1}}^{2}-p \int_{\Omega} K\left(\alpha_{\epsilon} P \delta_{\epsilon}\right)^{p-1+\epsilon} v^{2} \\
+O\left(\epsilon\left|v_{\epsilon}\right|_{H_{0}^{1}}^{2}\right) \\
=\left|v_{\epsilon}\right|_{H_{0}^{1}}^{2}-p \alpha_{\epsilon}^{p-1+\epsilon} K\left(a_{\epsilon}\right) \int_{\Omega}\left(\delta_{\epsilon}^{p-1+\epsilon}\right. \\
\left.+O\left(\delta_{\epsilon}{ }^{p-1+\epsilon} \theta_{\epsilon}\right)\right) v^{2}+o\left(|v|_{H_{0}^{1}}^{2}\right) \\
=\left|v_{\epsilon}\right|_{H_{0}^{1}}^{2}-p \alpha_{\epsilon}^{p-1+\epsilon} K\left(a_{\epsilon}\right) C_{0}^{\epsilon} \lambda_{\epsilon}^{\epsilon(n-2) / 2} \int_{\Omega} \delta_{\epsilon}^{p-1} v^{2} \\
+O\left(\int _ { \Omega } \left(\delta_{\epsilon}^{p-1+\epsilon}\right.\right. \\
\left.\left.-C_{0}^{\epsilon} \lambda_{\epsilon}^{\epsilon(n-2) / 2} \delta_{\epsilon}^{p-1}|v|^{2}\right)\right)+o\left(|v|_{H_{0}^{1}}^{2}\right)
\end{gathered}
$$

Using Remark 4, we find

$$
\begin{gathered}
Q_{\epsilon}(v, v)=Q_{0}(v, v)+o\left(|v|_{H_{0}^{1}}^{2}\right) \quad \text { with } \\
Q_{0}(v, v)=|v|_{H_{0}^{1}}^{2}-\int_{\Omega} \delta_{\epsilon}^{p-1} v^{2} .
\end{gathered}
$$

According to [1], $Q_{0}$ is coercive, that is, there exists some constant $\mathrm{c}>0$ independent of $\epsilon$, for $\square$ small enough, such that

$$
Q_{0}(v, v) \geq c|v|_{H_{0}^{1}}^{2} \quad \forall v \in E_{\left(a_{\epsilon}, \lambda_{\epsilon}\right)} .
$$

We also observe that

$$
\begin{gathered}
f_{\epsilon}(v)=\alpha_{\epsilon}^{p+\epsilon} \int_{\Omega} K\left(\delta_{\epsilon}{ }^{p+\epsilon}+O\left(\delta_{\epsilon}{ }^{p-1+\epsilon} \theta_{\epsilon}\right)\right) v \\
=\alpha_{\epsilon}^{p+\epsilon}\left[C_{0}^{\epsilon} \lambda_{\epsilon}^{\epsilon(n-2) / 2} \int_{\Omega} K \delta_{\epsilon}{ }^{p} v\right. \\
+O\left(\epsilon \int_{\Omega} K \log \left(1+\lambda_{\epsilon}^{2}\left|x-a_{\epsilon}\right|^{2}\right) \delta_{\epsilon}^{2}|v|\right. \\
\left.\left.+\int_{\Omega} \delta_{\epsilon}{ }^{p-1} \theta_{\epsilon}|v|\right)\right]
\end{gathered}
$$

The last equality follows from Remark 4 . Therefore we can write,

$$
\begin{gathered}
\text { with } B=B\left(a_{\epsilon}, d_{\epsilon}\right) \\
f_{\epsilon}(v)=O\left(\epsilon|v|_{H_{0}^{1}}+\int_{B} \delta_{\epsilon}^{p-1} \theta_{\epsilon}\left|v_{\epsilon}\right|+\int_{\mathbb{R}^{n} \backslash B} \delta_{\epsilon}{ }^{p}|v|\right) \\
f_{\epsilon}(v)=O\left(\left(\epsilon+\frac{\left|\nabla K\left(a_{\epsilon}\right)\right|}{\lambda_{\epsilon}^{2}}\right)|v|_{H_{0}^{1}}\right. \\
+|v|_{H_{0}^{1}}\left|\theta_{\epsilon}\right|_{L^{\infty}}\left(\int_{B} \delta_{\epsilon}^{\frac{8 n}{n^{2}-4}}\right)^{\frac{n+2}{2 n}} \\
\left.+\left|v_{\epsilon}\right|_{H_{0}^{1}}\left(\int_{\mathbb{R}^{n} \backslash B} \delta_{\epsilon}^{\frac{2 n}{n-2}}\right)^{\frac{n+2}{2 n}}\right)
\end{gathered}
$$

We notice that

$$
\int_{\mathbb{R}^{n} \backslash B} \delta_{\epsilon}^{2 n /(n-2)}=O\left(\frac{1}{\left(\lambda_{\epsilon} d_{\epsilon}\right)^{n}}\right)
$$

and

$$
\left(\int_{B} \delta_{\epsilon}^{\frac{8 n}{n^{2}-4}}\right)^{(n+2) / 2 n} \leq C\left\{\begin{array}{lll}
\frac{d_{\epsilon}^{(n-6) / 2}}{\lambda_{\epsilon}^{2}} & \text { if } & n>6 \\
\frac{\log \left(\lambda_{\epsilon} d_{\epsilon}\right)}{\lambda_{\epsilon}^{2}} & \text { if } & n=6 \\
\frac{1}{\lambda_{\epsilon}^{(n-2) / 2}} & \text { if } & n<6
\end{array}\right.
$$

Using (5), we obtain

$\left|f_{\epsilon}(v)\right| \leq C|v|_{H_{0}^{1}}$

$$
+C\left\{\begin{array}{ccc}
\left(\frac{\left|\nabla K\left(a_{\epsilon}\right)\right|}{\lambda_{\epsilon}}+\frac{1}{\left(\lambda_{\epsilon} d_{\epsilon}\right)^{n-2}}\right)|v|_{H_{0}^{1}} & \text { if } & n<6 \\
\left(\frac{\left|\nabla K\left(a_{\epsilon}\right)\right|}{\lambda_{\epsilon}}+\frac{\log \left(\lambda_{\epsilon} d_{\epsilon}\right)}{\left(\lambda_{\epsilon} d_{\epsilon}\right)^{4}}\right)|v|_{H_{0}^{1}} & \text { if } & n=6 \\
\left(\frac{\left|\nabla K\left(a_{\epsilon}\right)\right|}{\lambda_{\epsilon}}+\frac{1}{\left(\lambda_{\epsilon} d_{\epsilon}\right)^{(n+2) / 2}}\right)|v|_{H_{0}^{1}} \quad \text { if } & n>6
\end{array}\right.
$$

Combining (13), (14) and (15), we obtain the desired estimate. 


\section{Proof of Theorem}

Let us start by proving the following crucial result :

\section{Proposition 6}

Let $u_{\epsilon}$ satisfying the assumption of the theorem. Then,

$$
\begin{gathered}
\left|\alpha_{\epsilon} c_{1} \frac{H\left(a_{\epsilon}, a_{\epsilon}\right)}{\lambda_{\epsilon}^{n-2}}-\alpha_{\epsilon} \frac{c_{3}}{n^{2}} \frac{\Delta K\left(a_{\epsilon}\right)}{K\left(a_{\epsilon}\right) \lambda_{\epsilon}^{2}}+\alpha_{\epsilon} c_{2} \varepsilon\right| \leq c\left(\epsilon^{2}+\frac{1}{\lambda_{\epsilon}^{3}}+\right. \\
\left.|v|_{H_{0}^{1}}^{2}\right)+ \begin{cases}\frac{\log \left(\lambda_{\epsilon} d_{\epsilon}\right)}{\left(\lambda_{\epsilon} d_{\epsilon}\right)^{n}} & (n \geq 4) \\
\frac{1}{\left(\lambda_{\epsilon} d_{\epsilon}\right)^{2}} & (n=3)\end{cases}
\end{gathered}
$$

where $a_{\epsilon}, \lambda_{\epsilon}$ and $d_{\epsilon}=\left(a_{\epsilon}, \partial \Omega\right)$ are given in (7) and $c_{1}, c_{2}, c_{3}$ are positive constants defined by

$$
\begin{gathered}
c_{1}=c_{0}^{\frac{2 n}{n-2}} \int_{\mathbb{R}^{n}} \frac{d x}{\left(1+|x|^{2}\right)^{(n+2) / 2}} \\
c_{2}=\frac{n-2}{2} c_{0}^{\frac{2 n}{n-2}} \int_{\mathbb{R}^{n}} \log \left(1+|x|^{2}\right) \frac{|x|^{2}-1}{\left(1+|x|^{2}\right)^{n+1}} d x
\end{gathered}
$$

and

$$
c_{3}=c_{0}^{\frac{2 n}{n-2}} \int_{\mathbb{R}^{n}} \frac{|x|^{2}}{\left(1+|x|^{2}\right)^{n}} d x .
$$

\section{Proof.}

Multiplying $\left(P_{\epsilon}\right)$ by $\lambda_{\epsilon} \frac{\partial P \delta_{\epsilon}}{\partial \lambda} \quad$ and integrating on $\Omega$,

$$
\begin{gathered}
0=-\int_{\Omega} \Delta u_{\epsilon} \lambda_{\epsilon} \frac{\partial P \delta_{\epsilon}}{\partial \lambda}-\int_{\Omega} K u_{\epsilon}^{p+\epsilon} \lambda_{\epsilon} \frac{\partial P \delta_{\epsilon}}{\partial \lambda} \\
=\int_{\Omega} \nabla\left(\alpha_{\epsilon} P \delta_{\epsilon}+v_{\epsilon}\right) \nabla\left(\lambda_{\epsilon} \frac{\partial P \delta_{\epsilon}}{\partial \lambda}\right)-\int_{\Omega} K\left(\alpha_{\epsilon} P \delta_{\epsilon}\right. \\
\left.+v_{\epsilon}\right)^{p+\epsilon} \lambda_{\epsilon} \frac{\partial P \delta_{\epsilon}}{\partial \lambda} \\
=\alpha_{\epsilon} \int_{\Omega} \delta_{\epsilon}^{p} \lambda_{\epsilon} \frac{\partial P \delta_{\epsilon}}{\partial \lambda}-\int_{\Omega} K\left[\left(\alpha_{\epsilon} P \delta_{\epsilon}\right)^{p+\epsilon}\right. \\
+(p+\epsilon)\left(\alpha_{\epsilon} P \delta_{\epsilon}\right)^{p-1+\epsilon} v \\
+O\left(\delta_{\epsilon}^{p-2+\epsilon}\left|v_{\epsilon}\right|^{2}\right. \\
\left.\left.+\left|v_{\epsilon}\right|^{p+\epsilon}\right)\right] \lambda_{\epsilon} \frac{\partial P \delta_{\epsilon}}{\partial \lambda}
\end{gathered}
$$

We estimate each term of the right hand side in (17). First, we have

$$
\int_{\Omega} \delta_{\epsilon}^{p} \lambda_{\epsilon} \frac{\partial P \delta_{\epsilon}}{\partial \lambda}=\int_{\Omega} \delta_{\epsilon}^{p} \lambda_{\epsilon} \frac{\partial \delta_{\epsilon}}{\partial \lambda}-\int_{\Omega} \delta_{\epsilon}^{p} \lambda_{\epsilon} \frac{\partial \theta_{\epsilon}}{\partial \lambda}
$$

whence

$$
\begin{gathered}
\int_{\Omega} \delta_{\epsilon}^{p} \lambda_{\epsilon} \frac{\partial P \delta_{\epsilon}}{\partial \lambda} \\
=\int_{\mathbb{R}^{n}} \delta_{\epsilon}^{p} \lambda_{\epsilon} \frac{\partial \delta_{\epsilon}}{\partial \lambda}-\int_{\mathbb{R}^{n} \backslash \Omega} \delta_{\epsilon}^{p} \lambda_{\epsilon} \frac{\partial \delta_{\epsilon}}{\partial \lambda}-\int_{B} \delta_{\epsilon}^{p} \lambda_{\epsilon} \frac{\partial \theta_{\epsilon}}{\partial \lambda} \\
-\int_{\Omega \backslash B} \delta_{\epsilon}^{p} \lambda_{\epsilon} \frac{\partial \theta_{\epsilon}}{\partial \lambda} \\
=O\left(\frac{1}{\left(\lambda_{\epsilon} d_{\epsilon}\right)^{n}}\right)-\lambda_{\epsilon} \frac{\partial \theta_{\epsilon}}{\partial \lambda}\left(a_{\epsilon}\right) \int_{B} \delta_{\epsilon}^{p} \\
+O\left(\lambda_{\epsilon} \int_{B} \delta_{\epsilon}^{p} \mid x\right. \\
\left.-\left.a_{\epsilon}\right|^{2} \sup _{B}\left|D_{x}^{2} \frac{\partial \theta_{\epsilon}}{\partial \lambda}\right|\right)
\end{gathered}
$$

with $\quad \mathrm{B}=\left(\mathrm{a}_{\square}, \mathrm{d}_{\square}\right)$. According to [9], we have

$$
\begin{gathered}
\lambda_{\epsilon} \frac{\partial \theta_{\epsilon}}{\partial \lambda}\left(a_{\epsilon}\right)=-\frac{n-2}{2} \frac{c_{0}}{\lambda_{\epsilon}^{(n-2) / 2}} H\left(a_{\epsilon}, a_{\epsilon}\right) \\
+O\left(\frac{1}{\lambda_{\epsilon}^{(n+2) / 2} d_{\epsilon}^{n}}\right)
\end{gathered}
$$

and

$$
\sup _{B}\left|D_{x}^{2} \frac{\partial \theta_{\epsilon}}{\partial \lambda}\right|=O\left(\frac{1}{\lambda_{\epsilon}^{n / 2} d_{\epsilon}^{n}}\right)
$$

Therefore, estimating the integrals we obtain

$$
\begin{aligned}
\int_{\Omega} \delta_{\epsilon}^{p} \lambda_{\epsilon} \frac{\partial P \delta_{\epsilon}}{\partial \lambda}= & \frac{n-2}{2} c_{1} \frac{H\left(a_{\epsilon}, a_{\epsilon}\right)}{\lambda_{\epsilon}^{n-2}} \\
& +O\left(\frac{\log \left(\lambda_{\epsilon} d_{\epsilon}\right)}{\left(\lambda_{\epsilon} d_{\epsilon}\right)^{n}}\right)
\end{aligned}
$$

Secondly, we compute

$$
\begin{gathered}
\int_{\Omega} K\left(P \delta_{\epsilon}\right)^{p+\epsilon} \lambda_{\epsilon} \frac{\partial P \delta_{\epsilon}}{\partial \lambda} \\
=\int_{\Omega} K\left[\delta_{\epsilon}^{p+\epsilon}-(p+\epsilon) \delta_{\epsilon}^{p-1+\epsilon} \theta_{\epsilon}\right. \\
\left.+O\left(\theta_{\epsilon}^{2} \delta_{\epsilon}^{p-2+\epsilon}+\theta_{\epsilon}^{p+\epsilon}\right)\right] \lambda_{\epsilon} \frac{\partial P \delta_{\epsilon}}{\partial \lambda} \\
=\int_{B} K \delta_{\epsilon}^{p+\epsilon} \lambda_{\epsilon} \frac{\partial \delta_{\epsilon}}{\partial \lambda} \\
-\int_{B} K \delta_{\epsilon}^{p+\epsilon} \lambda_{\epsilon} \frac{\partial \theta_{\epsilon}}{\partial \lambda}-(p \\
+\int_{\Omega} K \delta_{\epsilon}^{p-1+\epsilon} \theta_{\epsilon} \lambda_{\epsilon} \frac{\partial \delta_{\epsilon}}{\partial \lambda} \\
+\int_{\Omega} \theta_{\epsilon}^{2} \delta_{\epsilon}^{p-1+\epsilon}+\int_{\Omega} \delta_{\epsilon}^{p-1+\epsilon} \theta_{\epsilon}\left|\lambda_{\epsilon} \frac{\partial \theta_{\epsilon}}{\partial \lambda}\right|+\int_{\Omega} \theta_{\epsilon}^{p+\epsilon} \delta_{\epsilon}+ \\
\left.\frac{\lambda_{\epsilon}{ }^{p(n-2)}}{\left(\lambda_{\epsilon} d_{\epsilon}\right)^{n}}\right)
\end{gathered}
$$


and we have to estimate each term of the right hand side of (18). Using the fact that $\lambda_{\epsilon} \frac{\partial \delta_{\epsilon}}{\partial \lambda}=\frac{n-2}{2}\left(\frac{1-\lambda_{\epsilon}^{2}\left|x-a_{\epsilon}\right|^{2}}{1+\lambda_{\epsilon}^{2}\left|x-a_{\epsilon}\right|^{2}}\right) \delta_{\epsilon}$ we derive that

$$
\begin{aligned}
& \int_{B} K \delta_{\epsilon}{ }^{p+\epsilon} \lambda_{\epsilon} \frac{\partial \delta_{\epsilon}}{\partial \lambda} \\
& =\frac{n-2}{2} \lambda_{\epsilon}^{\frac{\epsilon(n-2)}{n}} K\left(a_{\epsilon}\right) c_{0}^{p+1+\epsilon} \int_{\mathbb{R}^{n}} \frac{1}{\left(1+|x|^{2}\right)^{n+\frac{\epsilon(n-2)}{n}} \frac{1-|x|^{2}}{1+|x|^{2}} d x} \\
& +O\left(\frac{\lambda_{\epsilon}^{\frac{\epsilon(n-2)}{n}}}{\left(\lambda_{\epsilon} d_{\epsilon}\right)^{n}}\right) \\
& =\lambda_{\epsilon}^{\frac{\epsilon(n-2)}{n}}\left(c_{2} K\left(a_{\epsilon}\right) \epsilon-\alpha_{\epsilon} \frac{c_{3}}{n^{2}} \frac{\Delta K\left(a_{\epsilon}\right)}{\lambda_{\epsilon}^{2}}+O\left(\epsilon^{2}+\frac{1}{\lambda_{\epsilon}^{3}}\right)\right)+ \\
& O\left(\frac{\lambda_{\epsilon}}{\left(\lambda_{\epsilon} d_{\epsilon}\right)^{n}}\right)
\end{aligned}
$$

For the other terms in (19), we write

$$
\begin{aligned}
& \int_{B} K \delta_{\epsilon}^{p+\epsilon} \lambda_{\epsilon} \frac{\partial \theta_{\epsilon}}{\partial \lambda}=K\left(a_{\epsilon}\right) \lambda_{\epsilon} \frac{\partial \theta_{\epsilon}}{\partial \lambda}\left(a_{\epsilon}\right) \int_{B} \delta_{\epsilon}^{p+\epsilon} \\
& +O\left(\int_{B} \delta_{\epsilon}^{p+\epsilon} \frac{\left|x-a_{\epsilon}\right|^{2}}{\lambda_{\epsilon}^{(n-2) / 2} d_{\epsilon}^{n}}\right) \\
& =\frac{n-2}{2} K\left(a_{\epsilon}\right) c_{0}^{p+1+\epsilon} \frac{H\left(a_{\epsilon}, a_{\epsilon}\right)}{\lambda_{\epsilon}^{(n-2) / 2}} \int_{B}\left(\frac{\lambda_{\epsilon}}{1+\lambda_{\epsilon}^{2}\left|x-a_{\epsilon}\right|^{2}}\right)^{(p+\epsilon)(n-2) / 2} \\
& +O\left(\frac{\lambda_{\epsilon}^{\epsilon(n-2) / 2} \log \left(\lambda_{\epsilon} d_{\epsilon}\right)}{\left(\lambda_{\epsilon} d_{\epsilon}\right)^{n}}\right) \\
& =\frac{n-2}{2} c_{1} K\left(a_{\epsilon}\right) \frac{H\left(a_{\epsilon}, a_{\epsilon}\right)}{\lambda_{\epsilon}^{n-2}} \lambda_{\epsilon}^{\epsilon(n-2) / 2} \\
& +O\left(\frac{\lambda_{\epsilon}^{\epsilon(n-2) / 2} \log \left(\lambda_{\epsilon} d_{\epsilon}\right)}{\left(\lambda_{\epsilon} d_{\epsilon}\right)^{n}}\right) . \\
& \int_{B} K \delta_{\epsilon}^{p-1+\epsilon} \theta_{\epsilon} \lambda_{\epsilon} \frac{\partial \delta_{\epsilon}}{\partial \lambda} \\
& =\theta_{\epsilon}\left(a_{\epsilon}\right) K\left(a_{\epsilon}\right) \int_{B} \delta_{\epsilon}^{p-1+\epsilon} \lambda_{\epsilon} \frac{\partial \delta_{\epsilon}}{\partial \lambda} \\
& +O\left(\int_{B} \delta_{\epsilon}^{p+\epsilon} \frac{\left|x-a_{\epsilon}\right|^{2}}{\lambda_{\epsilon}^{(n-2) / 2} d_{\epsilon}^{n}}\right) \\
& \int_{B} K \delta_{\epsilon}^{p-1+\epsilon} \theta_{\epsilon} \lambda_{\epsilon} \frac{\partial \delta_{\epsilon}}{\partial \lambda}=\frac{n-2}{2} K\left(a_{\epsilon}\right) c_{1} \frac{H\left(a_{\epsilon}, a_{\epsilon}\right)}{\lambda_{\epsilon}^{n-2}} \lambda_{\epsilon}^{\epsilon(n-2) / 2}+ \\
& O\left(\frac{\lambda_{\epsilon}^{\epsilon(n-2) / 2} \log \left(\lambda_{\epsilon} d_{\epsilon}\right)}{\left(\lambda_{\epsilon} d_{\epsilon}\right)^{n}}\right) \text {. }
\end{aligned}
$$

(19), (20), (21) and additional integral estimates of the same type provide us with the expansion

$$
\begin{aligned}
\int_{\Omega} K\left(P \delta_{\epsilon}\right)^{p+\epsilon} \lambda_{\epsilon} & \frac{\partial P \delta_{\epsilon}}{\partial \lambda} \\
& =\frac{n-2}{2} \lambda_{\epsilon}^{\epsilon(n-2) / 2}\left[c_{2} K\left(a_{\epsilon}\right) \epsilon\right. \\
& \left.+2 c_{1} K\left(a_{\epsilon}\right) \frac{H\left(a_{\epsilon}, a_{\epsilon}\right)}{\lambda_{\epsilon}^{n-2}}-c_{3} \frac{\Delta K\left(a_{\epsilon}\right)}{\lambda_{\epsilon}^{2}}\right] \\
& +O\left(c_{3}+\frac{1}{\lambda_{\epsilon}^{3}}+\frac{\log \left(\lambda_{\epsilon} d_{\epsilon}\right)}{\left(\lambda_{\epsilon} d_{\epsilon}\right)^{n}}\right. \\
& \left.+\frac{1}{\left(\lambda_{\epsilon} d_{\epsilon}\right)^{2}} \quad(\text { if } n=3)\right)
\end{aligned}
$$

We note that

$\int_{\Omega} K\left(P \delta_{\epsilon}\right)^{p-1+\epsilon} v_{\epsilon} \lambda_{\epsilon} \frac{\partial P \delta_{\epsilon}}{\partial \lambda}$

$$
=\int_{\Omega} K\left(\delta_{\epsilon}^{p-1+\epsilon}\right.
$$$$
\left.+O\left(\theta_{\epsilon}^{p-1+\epsilon}+\delta_{\epsilon}^{p-2+\epsilon} \theta_{\epsilon}\right)\right) v_{\epsilon} \lambda_{\epsilon} \frac{\partial P \delta_{\epsilon}}{\partial \lambda}
$$$$
=\int_{\Omega} K \delta_{\epsilon}^{p-1+\epsilon} v_{\epsilon} \lambda_{\epsilon} \frac{\partial \delta_{\epsilon}}{\partial \lambda}
$$$$
-\int_{\Omega} K \delta_{\epsilon}^{p-1+\epsilon} v_{\epsilon} \lambda_{\epsilon} \frac{\partial \theta_{\epsilon}}{\partial \lambda}
$$$$
-O\left(\int_{\Omega} \delta_{\epsilon}^{p-1+\epsilon}\left|v_{\epsilon}\right| \theta_{\epsilon}\right)
$$$$
=\int_{\Omega} K\left(\delta_{\epsilon}\right)^{p-1+\epsilon} v_{\epsilon} \lambda_{\epsilon} \frac{\partial \delta_{\epsilon}}{\partial \lambda}
$$

$-O\left(\frac{\lambda_{\epsilon}^{\epsilon(n-2)}\left|v_{\epsilon}\right|_{H_{0}^{1}}}{\left(\lambda_{\epsilon} d_{\epsilon}^{2}\right)^{(n-2) / 2}}\left(\int_{\Omega} \delta_{\epsilon} \frac{8 n}{n^{2}-4}\right)^{(n+2) / 2}\right)$

$+O\left(\lambda_{\epsilon}^{\epsilon(n-2)}\left|v_{\epsilon}\right|_{H_{0}^{1}}\left(\int_{\Omega} \delta_{\epsilon}^{2 n /(n-2)}\right)^{(n+2) /(2 n)}\right)$

Using (15) we find

$$
\begin{array}{r}
\int_{\Omega} K\left(P \delta_{\epsilon}\right)^{p-1+\epsilon} v_{\epsilon} \lambda_{\epsilon} \frac{\partial P \delta_{\epsilon}}{\partial \lambda}=\int_{\Omega} K \delta_{\epsilon}^{p-1+\epsilon} v_{\epsilon} \lambda_{\epsilon} \frac{\partial \delta_{\epsilon}}{\partial \lambda}+ \\
O\left(\frac{\lambda_{\epsilon}^{\frac{\epsilon(n-2)}{2}\left|v_{\epsilon}\right|_{1}^{1}}}{\left(\lambda_{\epsilon} d_{\epsilon}\right)^{\frac{(n+2)}{2}}}\right)+O\left(\lambda _ { \epsilon } ^ { \frac { \epsilon ( n - 2 ) } { 2 } } | v _ { \epsilon } | _ { H _ { 0 } ^ { 1 } } \left[\frac{1}{\left(\lambda_{\epsilon} d_{\epsilon}\right)^{n-2}}(\text { if } n<\right.\right. \\
\left.\left.6)+\frac{\log \left(\lambda_{\epsilon} d_{\epsilon}\right)}{\left(\lambda_{\epsilon} d_{\epsilon}\right)^{4}}(\text { if } n=6)+\frac{1}{\left(\lambda_{\epsilon} d_{\epsilon}\right)^{\frac{(n+2)}{2}}}(\text { if } n>6)\right]\right)
\end{array}
$$

We also have, using Remark 4 


$$
\begin{aligned}
\int_{\Omega} K\left(\delta_{\epsilon}\right)^{p-1+\epsilon} v_{\epsilon} & \lambda_{\epsilon} \frac{\partial \delta_{\epsilon}}{\partial \lambda} \\
& =\lambda_{\epsilon}^{\frac{\epsilon(n-2)}{2}} c_{0}^{\epsilon} \int_{\Omega} K \delta_{\epsilon}^{p-1} v_{\epsilon} \lambda_{\epsilon} \frac{\partial \delta_{\epsilon}}{\partial \lambda} \\
& +\int_{\Omega} K\left(\delta_{\epsilon}^{p-1+\epsilon}\right. \\
& \left.-c_{0}^{\epsilon} \lambda_{\epsilon}^{\frac{\epsilon(n-2)}{2}} \delta_{\epsilon}^{p-1}\right) v_{\epsilon} \frac{\partial \delta_{\epsilon}}{\partial \lambda} \\
& =O\left(\epsilon \int_{\Omega} K \log (1\right. \\
& \left.\left.+\lambda_{\epsilon}^{2}\left|x-a_{\epsilon}\right|^{2}\right) \delta_{\epsilon}^{p}\left|v_{\epsilon}\right|\right)
\end{aligned}
$$

whence

$$
\begin{array}{cr}
\int_{\Omega} K\left(\delta_{\epsilon}\right)^{p-1+\epsilon} v_{\epsilon} \lambda_{\epsilon} \frac{\partial \delta_{\epsilon}}{\partial \lambda} & =O\left(\epsilon\left|v_{\epsilon}\right|_{H_{0}^{1}}\right)= \\
O(\epsilon) . & (25)
\end{array}
$$

Noticing that in addition $\lambda_{\epsilon} \frac{\partial P \delta_{\epsilon}}{\partial \lambda}=O\left(\delta_{\epsilon}\right)$ and

$$
\begin{aligned}
& \int_{\Omega} \delta_{\epsilon}^{p-1+\epsilon}\left|v_{\epsilon}\right|^{2}=O\left(\lambda_{\epsilon}^{\epsilon(n-2) / 2}\left|v_{\epsilon}\right|_{H_{0}^{1}}^{2}\right) . \\
& \int_{\delta<\left|v_{\epsilon}\right|}\left|v_{\epsilon}\right|^{p+\epsilon} \delta_{\epsilon}=O\left(\lambda_{\epsilon}^{\epsilon(n-2) / 2}\left|v_{\epsilon}\right|_{H_{0}^{1}}^{p+1}\right) .
\end{aligned}
$$

(18), (23), (24),(25), (26), (27) and Lemmas 3 and 5 prove Proposition 6.

We are know able to prove the theorem.

\section{Proof of Theorem 1}

Arguing by contradiction, let us suppose that $\left(P_{\epsilon}\right)$ has a solution $u_{\epsilon}$ as stated in the theorem. From Proposition 6, we have

$$
\begin{gathered}
\alpha_{\epsilon} c_{1} \frac{H\left(a_{\epsilon}, a_{\epsilon}\right)}{\lambda_{\epsilon}^{n-2}}-\alpha_{\epsilon} \frac{c_{3}}{n^{2}} \frac{\Delta K\left(a_{\epsilon}\right)}{K\left(a_{\epsilon}\right) \lambda_{\epsilon}^{2}}+\alpha_{\epsilon} c_{2} \epsilon=o\left(\epsilon+\frac{1}{\lambda_{\epsilon}^{2}}+\right. \\
\left\{\begin{array}{l}
\frac{1}{\left(\lambda_{\epsilon} d_{\epsilon}\right)^{n-2}}(\text { if } n \geq 4) \\
\frac{1}{\lambda_{\epsilon} d_{\epsilon}} \quad(\text { if } n=4)
\end{array}\right)
\end{gathered}
$$

Notice that $H\left(a_{\epsilon}, a_{\epsilon}\right) \sim d_{\epsilon}^{n-2}$ if $d_{\epsilon} \rightarrow 0$ as $\epsilon \rightarrow 0$ and $H\left(a_{\epsilon}, a_{\epsilon}\right) \geq c>0$ as $\epsilon \rightarrow 0$ if $d_{\epsilon} \nrightarrow 0$ as $\epsilon \rightarrow 0$.

For $n=3$, it follows from (28) that

$$
c_{1} \frac{H\left(a_{\epsilon}, a_{\epsilon}\right)}{\lambda_{\epsilon}}+c_{2} \epsilon=o\left(\epsilon+\frac{1}{\lambda_{\epsilon}}\right)
$$

which is a contradiction.

For $n=4$ it follows from (28) that

$c_{1} \frac{H\left(a_{\epsilon}, a_{\epsilon}\right)}{\lambda_{\epsilon}^{2}}-\frac{c_{3}}{16} \frac{\Delta K\left(a_{\epsilon}\right)}{K\left(a_{\epsilon}\right) \lambda_{\epsilon}^{2}}+c_{2} \epsilon=O\left(\epsilon+\frac{1}{\lambda_{\epsilon}^{2}}+\frac{1}{\left(\lambda_{\epsilon} d_{\epsilon}\right)^{2}}\right)$

which is a contradiction with assumption (ii) of the theorem.

For $n \geq 5$, it follows from (28) that

$$
-\frac{c_{3}}{n^{2}} \frac{\Delta K\left(a_{\epsilon}\right)}{K\left(a_{\epsilon}\right) \lambda_{\epsilon}^{2}}+c_{2} \epsilon=O\left(\epsilon+\frac{1}{\lambda_{\epsilon}^{2}}\right)
$$

also leads to a contradiction with assumption (iii) .

\section{References}

[1] A. Bahri, Critical point at infinity in some variational problems, Pitman Res. Notes Math. Ser. 182, Longman Sci. Tech. Harlow 1989.

[2] A. Bahri and J. M. Coron, On a nonlinear elliptic equation involving the Sobolev exponent : the effect of the topology of the domain, Comm. Pure Appl. Math. 41 (1988), 253-294

[3] A. Bahri, Y.Y. Li and O. Rey, On a variational problem with lack of compactness: the topological effect of the critical points at infinity, Calc. Var. and Part. Diff. Equ. 3 (1995), 67-94.

[4] M. Ben Ayed, K. El Mehdi, M. Grossi and O. Rey, A Nonexistence result of single peaked solutions to a supercritical nonlinear problem, Comm. Contenporary Math., 2 (2003), 179-195.

[5] M. Ben Ayed, K. Ould Bouh, Nonexistence results of sign-changing solutions to a supercritical nonlinear problem, Comm. Pure Applied Anal, 5 (2007), 1057-1075.

[6] M. Del Pino, P. Felmer and M. Musso, Two bubles solutions in the supercritical Bahri-Coron's problem, Calc. Var. Part. Diff. Equat., 16 (2003), 113-145.

[7] Z. C. Han, Asymptotic approach to singular solutions for nonlinear elliptic equations involving critical Sobolev exponent, Ann. Inst. Henri Poincare (Analyse non-linear) 8(1991),159-174.

[8] K. Ould Bouh, Nonexistence result of sign-changing solutions for a supercritical problem of the scalar curvature type, Advance in Nonlinear Studies (ANS), 12 (2012), 149-171.

[9] O. Rey, The role of Green's function in a nonlinear elliptic equation involving critical Sobolev exponent, J. Funct. Anal. 89 (1990), 1-52.

[10] O. Rey, The topological impact of critical points at infinity in a variational problem with lack of compactness : the dimension 3, Adv. Diff. Equ. 4 (1999), 581-616. 